\title{
NI AQUÍ, NI ALLÁ. L'IMMIGRAZIONE FEMMINILE DALL'ECUADOR
}

\author{
CHIARA PAGNOTTA
}

Università di Genova (Italia).

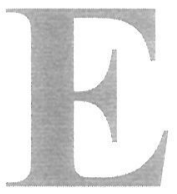

\section{RESUMEN}

ste trabajo se aproxima a las subjeividades de las mujeres ecuatorianas que han migrado desde el Ecuador hasta Génova (Italia) y se centra en los factores clave para la consecución de una mejor calidad de vida: trabajo, vivienda e integración. La realidad de la Unión Europea y de Italia en materia de política migratoria no está dando respuesta a estas cuestiones que, más allá de estar vinculadas a los derechos humanos, son situaciones de vivencia diaria.

Palabras clave: Migración femenina, políticas migratorias, Italia, Génova.

Da noi, dire cultura... non sai neanche cosa vuol dire cultura, perché non...

La cultura è quello che hai studiato di più là.

Qua la cultura è un modo di vivere, è importante. Mai (prima) ti eri messa a pensare qual era la tua cultura, dove sei nata, quali sono le tue origini.

Senti la mancanza del tuo paese, quando vai là certe cosa le vedi strane perché hai imparato un po' ad essere italiana.

Allora, sei qua e ti manca là, vai là e ti manca qua.... Ti viene una crisi perché pensi: "dove cavolo mi trovo?"

\section{INTRODUZIONE}

Queste parole sintetizzano chiaramente la natura dei processi migratori e delle dinamiche che li attraversano. La cultura e l'identità non appaiono come definiti a priori, ma come elementi che si costruiscono nel tempo, che si modificano secondo le esperienze di vita. 
Nell'ambito degli studi migratori, spesso si è portati a sottendere che l'identità del migrante sia, in qualche modo, predeterminata dalle origini e non un processo dinamico. Si tende a considerare il concetto di cultura immutabile basandosi sulla sovrapposizione di essa con l'etnia. Una simile concezione presenta risolto a priori il nodo fondamentale degli studi sulle migrazioni: il problema della produzione, riproduzione e trasformazione dell'identità dei migranti ${ }^{2}$. Proprio alcuni antropologi hanno duramente e recentemente criticato il concetto di multiculturalismo ${ }^{3}$.

A partire dal saggio di Edward Sayd, molti studiosi hanno rilevato come uno dei dati fondanti dei concetti di Occidente e di Europa sia la comune esperienza coloniale; essa, tralasciando l'aspetto storico, ha dato origine ad una idea immutabile di alterità e identità dei popoli extraeuropei a causa del lavoro di classificazione attuato dagli amministratori coloniali ${ }^{4}$.

L'immagine ricorrente dell'immigrato o dell'emigrato è quella di un soggetto che da un mondo, un cultura, un'identità si trasferisce verso un altro mondo, un'altra cultura e un'altra identità altrettanto impermeabile, che è possibile solamente accettare o rifiutare integralmente.

E' proprio l'adozione di una prospettiva di ricerca basata sulla soggettività dei migranti che ci permette di vedere come le forme di vita di tante donne di nazionalità ecuadoriane siano delle costruzioni sociali e non l'espressione di una presunta identità culturale esistente a priori.

Molti studi hanno concentrato l'attenzione sui grandi movimenti migratori di massa (ad es. la Grande Migrazione), ma nuove correnti storiografiche mettono in evidenza come accanto ad essi esista una mobilità di uomini e donne, interna ed esterna, che rappresenta un fattore di lunga durata dovuto all'industrializzazione ed all'urbanizzazione e determinato dalla riscrittura dei rapporti sociali tradizionali, caratteristico sia delle società di antico regime sia di quelle contemporanee ${ }^{5}$. L'immagine del continente europeo come sedentario

2 Sandro Mezzadra, Diritto di fuga, Migrazioni, cittadinanza, globalizzazione, Vcrona, Ombre corto, 2001, p.85.

3 Vedi Jean Loup Amscile, Logiche meticce. Antropologia dell'identità in Africa e altrove, Torino, bollati \& Borlinghieri, 1999; J. Clifford, I frutti puri impazziscono. Enografia, letteratura ed arte nel secolo XX, Torino, Bollati Borlinghieri, 1999.

4 Edward Said, Orientalismo. L'immagine europea dell'Occidente, Milano, Feltrinelli, 1999. V. anche Jean Loup Amselle, Logiche meticce, cit..

5 Vedi Angelina Arru, Josef Ehmer, Franco Ramella (a cura di), Migrazioni, "Quaderni Storici", anno XXXVI (2001), n. 106, fascicolo 1; Paola Corti, Storia delle migrazioni internazionali, Laterza, Roma-Bari, 2003; Donna Gabaccia, Emigranti, Le diaspore degli italiani dal Medioevo ad oggi, Einaudi, Torino, 2003; Yann Moulier Boutang, Dalla schiavitù al lavoro salariato, Manifestolibri, Roma 2003; Matteo Sanfilippo, Problemi di storiografia dell'emigrazione italiana, Sette città, Viterbo, 2002; Saskia Sassen, Migranti, coloni rifitgiati, Dall 'emigrazione di mas'sa alla fortezza Europa, Milano, Feltrinelli, 1999. 
risulta quindi errata. Nuovi studiosi mettono in discussione l'immagine, tramandata dalle scienze storico-economiche di tradizione marxista, di un età moderna in cui il lavoro salariato è libero, contrapposta ad un'epoca feudale dove la forma del lavoro è di tipo servile 6 . Dai loro studi appare evidente come, nel mondo contemporaneo, coesistano contemporaneamente forme di lavoro libero, semi-libero e servile e come le differenze non siano così marcate soprattutto per i migranti.

In questo saggio cercherò di collegare tra loro l'individuo ed il contesto, poiché ritengo che le due dimensioni siano indissociabili e che le azioni, le aspettative ed i desideri degli uomini, o delle donne, siano fortemente determinati dalle mutevoli interazioni con l'ambiente circostante. L'ipotesi che intendo sviluppare è che il progetto migratorio non sia limitato temporalmente al momento di lasciare il paese di origine, ma che piuttosto esso si sviluppi nel tempo attraverso alcune variabili, tra le quali ritengo fondamentali il viaggio, e l'insediamento in un novo paese, nonché le aspettative sviluppate. Ognuna di queste tappe non può essere analizzata come una variabile in sé, ma è costantemente rimodellata dall'ambiente in cui si trovano a vivere le donne ecuadoriane.

Al fine dell'analisi prenderò in esame la città di Genova e la popolazione delle donne ecuadoriane in città.

I gender's studies hanno messo in luce come molte delle categorie usate nell'analisi dei fenomeni migratori non siano appropriate per lo studio delle migrazioni femminili. Molte ricerche che appaiono neutre dal punto di vista del genere sono in realtà strutturate su un modello di migrante che è maschile 7 . Un modo per superare questo problema è l'uso dell'intervista orale, delle storie di vita. Tramite esse si può cogliere la natura dell'esperienza migratoria e le differenze tra le varie donne, avendo una visione olistica del loro vissuto personale. Le interviste rendono possibile, inoltre, cogliere la natura del contesto dall'interpretazione che ne danno gli attori.

Le testimonianze orali forse non aggiungono informazioni per l'analisi quantitativa, ma sono insostituibili nella comprensione di cosa l'esperienza migratoria abbia significato per coloro le quali lo hanno vissuto, di quali aspettative lo avevano investito, quali siano i

6 Moulier Boutang, Dalla schiavitù al lavoro salariato, cit.

7 Giovanna Campani, Genere, etnia e classe. Migrazioni al femminile tra esclusione ed identità, Pisa, ETS, 2000; bell hooks, Elogio del margine, Razza, sesso e mercato culturale, Milano, Feltrinelli, 1998; Giovanna Favaro, Maria Tognetti Bordogna, Donne dal mondo, strategie migratorie al femminile, Guerini Milano 1991; Mario Grasso, Donne senza confini. Immigrate in Italia tra marginalità ed emancipazione, Torino, L'Harmattan Italia, 1996; Eleonore Kofman, Annie Phizacklea, Parvati Raghuram, Rosemary Sales, Gender and international migration in Europe, Londra, Routlege, 2000; Iris Marion. Young, Le politiche della differenza, Milano, Feltrinelli, 1996. 
costi sociali ${ }^{8}$. Balza immediatamente agli occhi come il tempo di vita a Genova sia quasi esclusivamente il tempo del lavoro, per questo motivo ho deciso di considerare in modo particolare questo aspetto.

Il materiale che ho utilizzato è l'elaborazione delle storie di vita di tredici donne ecuadoriane che ho intervistato tra il 2002 ed il 2003 nella città di Genova. Si tratta di testimonianze dettagliate sulla storia delle singole donne, sulle motivazioni che le hanno spinte a lasciare l'Ecuador, sul vissuto a Genova e sulle proprie aspettative. L'analisi delle storie di vita è preceduta da un inquadramento generale del panorama migratorio italiano e genovese

\section{LA TERRA DI ARRIVO}

\subsection{Il contesto}

Nel corso degli ultimi 20-30 anni il volto dell'immigrazione in Italia è cambiato molto rapidamente a causa di mutamenti economici, sociali e politici avvenuti non unicamente nei paesi di origine. Oggi la presenza straniera sul territorio rappresenta poco meno che il $4 \%$ della popolazione residente. Con la Sanatoria del 2002, varata in coincidenza dell'ultima legge sull'immigrazione (Bossi-Fini), il numero degli stranieri in Italia è di circa $2400000^{\circ}$.Questi dati numerici si riferiscono, ovviamente, alla popolazione regolare.

Più che su una questione numerica, vorrei porre all'attenzione il cambiamento avvenuto per quanto riguarda il genere. Come afferma Reyneri, i tratti caratterizzanti l'immigrazione degli anni 50-60 individuano la figura tipo del migrante come il giovane, uomo, celibe o comunque senza coniuge ${ }^{10}$. In anni più recenti il panorama si è modificato.

Come si evince dalla tabella, dai paesi del Maghreb e dall'Egitto emigrano soprattutto uomini, ma la Tunisia, che nel 1992 era la seconda nazionalità presente, nel 2000 è diventata la sesta. L'Albania, che nel 1992 era al quinto posto con una migrazione in netta prevalenza maschile, ha accresciuto le presenze fino a diventare la seconda nazionalità con una prevalenza meno schiacciante degli uomini. Con gli anni sono cresciute le nazionalità come Romania, Cina e Jugoslavia, per le quali

8 Alessandro Portelli, Problemi di metodo, Sulla diversità della storia orale, in Cesare Bermani (a cura di), Introduzione alla storia orale. Storia, conservazione delle fonti e problemi di metodo, Roma, Odradek, 1999. p.154.

9 Caritas di Roma, Immigrazione, Dossier statistico 2003, Roma, Anterem, 2003

10 Emilio Reyneri, La catena migratoria, Bologna, Il Mulino, 1983, p.36 
risulta che siano entrambi i generi ad emigrare più o meno in numero eguale. Vi sono, inoltre, le nazionalità per cui sono le donne ad emigrare in grandi quantità: Filippine, Polonia e più recentemente il Perù. Non è completamente irrilevante che le nazionalità a maggioranza migratoria femminile siano quelle a netta prevalenza cattolica. Una supposta vicinanza culturale infatti facilita l'accettazione nelle nuove società e una rete di controllo sociale nel contesto di arrivo.

Tabella 1: Immigrazione extracomunitaria in Italia anni 1992, 1999, 2000

\begin{tabular}{|c|c|c|c|c|c|c|c|c|}
\hline \multicolumn{3}{|c|}{1992} & \multicolumn{3}{|c|}{1999} & \multicolumn{3}{|c|}{2000} \\
\hline PAESI & NUMERO & $\%$ MASCHI & PAESI & NUMERO & \% MASCHI & PAESI & NUMERO & \% MASCHI \\
\hline Marocco & 83.292 & 90,2 & Marocco & 128.297 & 73,2 & Marocco & 155.864 & 72,3 \\
\hline Tunisia & 41.547 & 91,0 & Albania & 87.595 & 63,8 & Albania & 133.018 & 66,0 \\
\hline Filipinne & 36.316 & 32,8 & Filippine & 59.074 & 32,9 & Filippine & 67.386 & 33,8 \\
\hline Jugoslavia & 26.717 & 62,9 & Cina & 41.237 & 53,9 & Romania & 61.212 & 51,1 \\
\hline Albania & 24.886 & 85,9 & Tunisia & 41.137 & 78,5 & Cina & 56.660 & 54,9 \\
\hline Senegal & 24.194 & 97,1 & Jugoslavia & 6.099 & 60,1 & Tunisia & 46.773 & 77,9 \\
\hline Egitto & 18.473 & 85,8 & Romania & 33.777 & 44,5 & Jugoslavia & 41.234 & 58,6 \\
\hline Cina & 15.776 & 60,2 & Senegal & 31.420 & 93,3 & Senegal & 40.890 & 92,9 \\
\hline Pollonia & 12.139 & 44,3 & Sri Lanka & 27.381 & 57,0 & Egitto & 34.042 & 82,1 \\
\hline Sri Lanka & 12.114 & 69,0 & Egitto & 23.811 & 79,3 & Sri Lanka & 31.991 & 57,3 \\
\hline Ghana & 11.303 & 69,0 & Peru & 23.637 & 31,4 & Polonia & 29.478 & 29,5 \\
\hline Brasile & 10.953 & 31,4 & Polonia & 23.258 & 30,9 & Peru & 29.074 & 31,9 \\
\hline
\end{tabular}

Fonte: elaborazione Istat su dati Ministero dell'Interno".

Appare necessario ricordare che le donne che si spostano in modo indipendente dal proprio compagno o dal padre sono sempre esistite, ma solo negli ultimi anni nuove correnti di studio gli hanno tributato loro il giusto riconoscimento, inserendo nello studio dei processi migratori anche la variabile di genere.

Per quanto riguarda l'Italia è interessante notare come fin dagli anni '70 essa sia meta di alcune migrazioni a carattere prevalentemente femminile. Mi riferisco in modo particolare alle donne eritree le quali, con il denaro guadagnato come colf in Italia, sostenevano la lotta di liberazione del proprio popolo. Il canale di entrata era rappresentato dai legami coloniali tra i due paesi, mentre per altre nazionalità era la Chiesa cattolica a fornire l'orizzonte conoscitivo comune che facilita gli spostamenti (Filippine, Polonia...) $)^{12}$.

12 Elena Filippo, Enrico Pugliese, Le donne nell'immigrazione in Campania, , in AA. VV. (a cura di), Inmigración femina en el sur de Europa, Revista de sociologia, papers n.60, Universidad autonoma de Barcellona, 2000, pp. 55-66, pp. 57-58 


\subsection{Genova terra anelata.}

Per quanto riguarda l'America Latina, Genova è sempre stata un delle mete privilegiate dell'esilio cileno (263 presenze nel 1988) ${ }^{13}$. Ad arrivare erano prevalentemente uomini che si inserivano in lavori legati all'attività portuale o edile.

Verso la metà degli anni '90 acquistano consistenza le migrazioni dal Perù ed in seguito dall'Ecuador, con una netta predominanza del genere femminile. Nel 1995 i peruviani sono la seconda comunità a Genova, con 447 presenze regolari, nel 1996 sono superati dagli ecuadoriani con 1073 presenze; i marocchini sono ancora la prima comunità con 1264 persone.

Il dossier statistico della Caritas del 1999 fotografa una situazione di equilibrio migratorio tra i differenti generi. All'interno dell'insieme della popolazione straniera, le donne costituiscono di circa il $49.2 \%{ }^{14}$. Analizziamo ora la situazione con maggior dettaglio.

Tabella 2: Nazionalità maggiormente presenti a Genova

\begin{tabular}{|c|c|c|c|c|c|c|c|c|c|}
\hline & \multicolumn{3}{|c|}{1998} & \multicolumn{3}{|c|}{1999} & \multicolumn{3}{|c|}{2000} \\
\hline & TOT & M & F & TOT & M & F & TOT & M & F \\
\hline Marocco & 1587 & 1252 & 335 & 1820 & 1450 & 370 & 2128 & 1661 & 467 \\
\hline Senegal & 797 & 762 & 35 & 363 & 319 & 44 & 1100 & 1037 & 63 \\
\hline Ex Jugoslavia & 320 & 184 & 136 & 316 & 173 & 143 & 315 & 169 & 146 \\
\hline San Marino & 303 & 155 & 148 & 288 & 148 & 140 & 275 & 141 & 134 \\
\hline Cile & 310 & 186 & 124 & 321 & 189 & 132 & 337 & 196 & 141 \\
\hline Ecuador & 1419 & 424 & 995 & 2343 & 742 & 1601 & 3048 & 993 & 2055 \\
\hline Iran & 223 & 130 & 93 & 219 & 126 & 93 & 227 & 130 & 97 \\
\hline India & 245 & 61 & 184 & 215 & 68 & 196 & 286 & 77 & 209 \\
\hline Perù & 1004 & 318 & 686 & 1169 & 391 & 778 & 1331 & 451 & 882 \\
\hline Sri Lanka & 472 & 279 & 193 & 507 & 282 & 225 & 560 & 316 & 244 \\
\hline Tunisia & 286 & 198 & 88 & 308 & 208 & 100 & 342 & 229 & 113 \\
\hline Cina & 410 & 210 & 200 & 211 & 240 & 229 & 588 & 300 & 288 \\
\hline Filippine & 227 & 75 & 152 & 246 & 85 & 161 & 272 & 90 & 182 \\
\hline Albania & 494. & 321 & 173 & 736 & 479 & 257 & 1099 & 694 & 405 \\
\hline
\end{tabular}

Fonte: Annuario Statistico del Comune di Genova, anni 1998, 1999, 2000, mie elaborazioni.

Come si evince dalla tabella, dal 1999 in poi gli ecuadoriani sono la prima comunità migrante a Genova. Sia dal Perù, sia dall'Ecuador arrivano in maggioranza le donne. L'ultima Sanatoria, coincidente con la nuova legge sull'immigrazione, la Bossi-Fini del 2002, censisce, sul territorio della provincia di Genova circa undicimila presenze. Se teniamo come punto di osservazione per l'analisi il genere, si può notare che il $70 \%$ delle richieste di regolarizzazione è fatta da donne, 
principalmente per lavori come colf e badanti; se invece guardiamo alla nazionalità come dato cardine, si può notare che il $50-60 \%$ delle richieste sono fatte da ecuadoriani ${ }^{15}$. Secondo il Console dell'Ecuador, la presenza di connazionali in città, tra regolari ed irregolari, è da situarsi intorno alle sedicimila unità.

\section{ES QUE AQUÍ NO HAY FUTURO}

Il percorso delle donne ecuadoriane a Genova appare motivato dal desiderio di riacquistare uno status perduto, attraverso la migrazione.

Come si evince dal paragrafo precedente, la migrazione dall'Ecuador è un fenomeno piuttosto recente e coincide con la progressiva proletarizzazione dei ceti medi avvenuta negli ultimi anni in quel paese. Tra il 1995 ed il 2000 l'Ecuador fu il paese dell'America latina che conobbe il più veloce impoverimento: il numero di poveri passò da 3,9 a 9,1 milioni ${ }^{16}$. Le cause furono molteplici: il conflitto armato col Perù nel 1995, il Niño nel 1997 e 1998, la crisi finanziaria e politica, il blocco dei depositi bancari... ${ }^{17}$

Tabella 3: Migrazione delle/degli ecuadoriane/i tra il 1992 e il 2000

\begin{tabular}{ccccccccc}
\hline $\mathbf{1 9 9 2}$ & $\mathbf{1 9 9 3}$ & $\mathbf{1 9 9 4}$ & $\mathbf{1 9 9 5}$ & $\mathbf{1 9 9 6}$ & $\mathbf{1 9 9 7}$ & $\mathbf{1 9 9 8}$ & $\mathbf{1 9 9 9}$ & gennaio-luglio 2000 \\
\hline 25900 & 30683 & 37349 & 33146 & 29780 & 30931 & 49176 & 108837 & 84323 \\
\hline
\end{tabular}

Fonte: Dirección Nacional Migración in Proyecto "Migración, comunicación y desarroyo" op.cit. cap. I

Come si può intuire dalla tabella, l'acutizzarsi della crisi economica spinge molti ecuadoriani a praticare la migrazione.

Le donne che ho incontrato provengono tutte da una classe media impoveritisi negli ultimi anni. Le biografie da me raccolte, descrivono maestre, impiegate di banca, segretarie spinte a partire perché gli stipendi percepiti non riuscivano più a soddisfare le loro necessità. Nel 1999, al fine di sanare l'economia del paese, i dipendenti pubblici non percepirono gli stipendi per alcuni mesi. Sono proprio questi settori sociali a praticare la migrazione. L'entrata in Europa è un investimento economico non indifferente (volo aereo, bolsa de viaje ${ }^{18}$,

15 Fonte: Questura di Genova.

16 Alberto Acosta, Ecuador: Denda externa y migración, una relación incestuosa, Texto para la Universidad de Genova, 30 abril 2002, 1, mia traduzione

17 Proyecto "Migración comunicación y desarrollo", El proceso migratorio de ecucadorianos a España, Quito, Centro de Investigaciones ciudad, cap. 2; AA. VV. Causas del reciente proceso migratorio ecuadoriano. "Cartilla sobre la migración", diciembre del 2002, n.3, pp. 6-7 mia traduzione.

18 Quantità di denaro richiesta dagli stati di destinazione per potervi soggionare temporaneamente come turista. 
capitale necessario in caso di inattività lavorativa...) e generalmente gli ecuadoriani, uomini e donne, si rivolgono ai coyotes ${ }^{19}$ per ottenere un prestito lasciando i beni di famiglia a garanzia di un futuro pagamento.

La crescita esponenziale del fenomeno migratorio ha diversificato le direzioni, le persone che decidono di spostarsi. Mentre fino al 1997 ed emigrare erano prevalentemente gli uomini, recentemente la situazione si è capovolta. Anche le destinazioni sono cambiate, mentre fino al 1997 si andava principalmente negli Stati Uniti, in seguito ci si sposta verso la Spagna $(61,5 \%$ nel 2000$)$, negli USA $(19,5 \%)$ e in Italia $(10,0 \%)^{20}$. Proprio per il tipo di sviluppo economico di cui parlerò più diffusamente in seguito, le donne che si dirigono negli ultimi due paesi si inseriscono in tipologie di lavoro riservate tipicamente alla manodopera femminile: colf e badanti. Questo accade nonostante esse siano-come per le donne da me incontrate-in possesso di una istruzione universitaria ed abbiano svolto in passato altri generi di lavoro.

Per alcune ecuadoriane la scelta migratoria e motivata dal desiderio di riconquistare individualmente un benessere perduto, per altre dalle necessità economiche nella propria famiglia. In tutte vi è comunque il desiderio più o meno latente di volere di più di quel che è nel proprio destino economico.

Non bisogna interpretare le motivazioni individuali o familiari che spingono alla migrazione come una scelta dicotomica; per le donne che ho incontrato coesistono entrambe le motivazioni. Le donne che lasciano l'Ecuador affidano la cura dei figli alla madre o alla sorella, fatto che implica l'assenza di rottura con la comunità di partenza, mentre chi si sposta per una scelta familiare mi ha fatto capire quanto sia ambigua il concetto di decisione collettiva, in quanto esso idealizza un ambito che non si basa su relazioni paritarie al suo interno; alcune donne si trovano a Genova quindi a causa di una decisione non completamente propria. La mia impressione, parlando con queste testimoni, è quella che esse non vogliano comunque proporsi in rottura con il paese di origine ed il loro porre l'accento sulle necessità economiche familiari abbia ai loro occhi anche una valenza giustificativa. I rischio sentito da queste donne è di essere considerate "avide"21.

19 Nome emblematico e popolare degli usurai.

20 Fonte: Gioconda Herrera, Ana Martinez, Género y migración en la region sur, Quito, Facultad Latinoamericana de Ciencias Sociales, 2000, mia elaborazione.

21 Alcune similitudini le ho trovate nel lavoro di Abdemalek Sayad, La doppia assenza. Dalle illusioni dell'emigrato alle sofferenze dell'immigrato, Milano, Raffaello Cortina, 2002. 


\section{ANTICHE O MODERNE SERVITU'?}

Prescindendo dalle differenze oggettive, che esistono, tra migrazioni interne e migrazioni internazionali, il modello produttivo, che ha il suo apice nella forma fordista della produzione industriale e la sua caduta in coincidenza della crisi economica degli anni '70-' 80 , vede i nuovi arrivati inserirsi in un mercato occupazionale, a carattere maschile, che ha nel lavoro di fabbrica la propria centralità. Principali beneficiari del patto fordista ${ }^{22}$ sono gli operai qualificati, denominati aristocrazia operaia, che in qualche modo sono integrati e difesi dal sistema dello Stato Sociale; una massa di non-integrati resta ai margini del sistema economico $^{23}$. Queste differenze sono ulteriormente rimarcate dalle differenze di sesso, razza ed etnia ${ }^{24}$. Attualmente il lavoro operaio in Europa non è affatto scomparso, ma è stato scalzato, dal ruolo egemone rivestito nella fase precedente, da figure produttive legate al differenti forme di lavoro; in specifico le forme di precarizzazione salariale riguardano alcuni gruppi più che altri; tra questi gli immigrati e le donne, sono loro che vanno a formare il moderno esercito di riserva determinato dalla divisione internazionale del lavoro ${ }^{25}$.

Wallerstein parla di etnicizzazione della forza lavoro ${ }^{26}$, cioè, determinati settori lavorativi appaiono esclusivo appannaggio di determinati gruppi e questo determina una teorizzazione alquanto caotica riguardo al fatto che esistano delle supposte propensioni culturali o biologiche nello svolgere determinate tipologie di lavori. Ad esempio, le donne migranti sarebbero naturalmente propense a fare le prostitute $o$ le colf. Le latinoamericane in genere, per l'innata e presunta dolcezza, sarebbero particolarmente richieste per la cura degli anziani.

Per quanto riguarda le ecuadoriane che vivono a Genova, il lavoro domestico rappresenta un imbuto lavorativo da cui è difficile uscire ${ }^{27}$.

L'inclusione subordinata delle donne ecuadoriana nei settori di cura alle persone ha la sua ragion d'essere sia nella affermazione di uno status

22 Per una spiegazione del patto fordista vedi Alain Birh, Dall assalto al cielo all alternativa, La crisi del movimento operaio europeo, Bfs, Pisa, 1995, cap. 2, 11 compromesso fordista, pp. $31-42$.

23 Vedi Asor Rosa, Le due società, Torino, Einaudi, 1977.

24 Alain Birh, Dall'assalto al cielo all'alternativa, La crisi del movimento operaio europeo, Bfs, Pisa, 1995, pp. 43-44.

25 Laura Oso, La inmigración femenina en Madrid y Lisboa: hacia una etnización del servicio doméstico y de las impresas de limpieza, in AA. VV. (a cura di), Inmigración femina en el sur de Europa, 183-207, p. 186.

26 Immanuel Wallerstein, Universalismo contro razzismo e sessismo: le tensioni ideologiche del capitalismo, in Etienne Balibar, Immanuel Wallerstein (a cura di), Razza Nazione Classe. Le idenità ambigue, Roma, Edizioni Associate, 1996, pp. 47-56, p. 52

27 Andrea Torre, Il contesto dell 'immigrazione femminile a Genova ed in Liguria, in Andrea Torre (a cura di), Non sono venuta a scoprire le scarpe. Voci di domne immigrate in Liguria, Dogliani, Sensibili alle foglic, 2001 , pp. 9-24, pp. 16-22. 
simbol, sia nel sistema dello stato sociale italiano.Per alcune persone appartenenti alle classi medio-elevate, l'affermazione del proprio benessere e del proprio successo individuale è dimostrato dall'avere una persona al proprio servizio ${ }^{28}$. Contemporaneamente, però, la carenza di servizi preposti all'aiuto alle famiglie con un anziano malato od un bambino fa sì che chi ne ha la possibilità si rivolga al settore privato per trovare assistenza. Una spiegazione della notevole crescita dei lavori di assistenza a Genova può essere proprio questa, anche alla luce del fatto che la città ha un tasso di invecchiamento molto elevato rispetto alla media europea ${ }^{29}$. Ciò che sembra favorire la presenza di donne latinoamericane nelle case altrui è una supposta vicinanza culturale o razziale.

I rapporti tra il datore di lavoro e la domestica ecuadoriana sono contrassegnati da un forte squilibrio di potere: le donne devono sempre essere a disposizione, non hanno orari di lavoro certi, le mansioni loro richieste sono le più disparate, la differenza linguistica, l'isolamento dato dal fatto di coabitare col proprio datore di lavoro, l'assenza di un permesso di soggiorno relega le ecuadoriane in una situazione di handicap. Non a caso, infatti, l'apprendimento dell'italiano è visto come una necessità prioritaria per imparare a difendersi nelle situazioni avverse.

Il fatto di vivere nel luogo in cui si lavora determina una situazione per cui si è sempre a disposizione per le mansioni che vengono richieste, e rimane come tempo per sé solamente uno o due pomeriggi la settimana perché tutto ciò è ritenuto ovvio dal datore di lavoro.

Le donne che ho intervistato mi hanno raccontato che al minimo segno di contrarietà verso lo strapotere del datore di lavoro vengono accusate di non volere lavorare.

Insomma, queste donne sono qui per essere subordinate. Una simile situazione rientra pienamente nei parametri codificati da Ceschi e Mazzoni per definire cosa sia oggi il lavoro servile in Italia ${ }^{30}$. Le ecuadoriane si rivolgono ai signori-padroni (definizione riferitami dalle stesse domestiche), mentre i datori di lavoro si rivolgono alle dipendenti dandogli del tu e chiamandole col nome di battesimo, il più delle volte storpiato. Un fatto simile, che all'apparenza può passare inosservato,

28 Torre, Il contesto dell 'immigrazione femminile a Genova ed in Liguria, cit. p. 17.

29 Ibidem, 16.

30 Sebastiano Ceschi, Martino Mazzoni, Le forme dello sfruttamento servile e paraschiavistico nel mondo del lavoro, in Francesco Carchedi, Giovanni Matura, Enrico Pugliese (a cura di), ll lavoro servile e le move servitu, Milano, Franco Angeli, 2003, pp. 27-45, p. 83. 
in realtà rivela una logica simbolica di dominazione ${ }^{31}$. Hannah Arendt afferma che molte attività lavorative del mondo moderno rientrino nella classificazione di servitù, anche se in linea teorica un simile modello produttivo si pensa sia applicabile unicamente alle società antiche. Come nella Grecia antica, le attività pubbliche sono riservate al genere maschile, mentre donne e schiavi sono relegati tra le mura domestiche ${ }^{32}$. Le donne migranti, come afferma Giovanna Campani, si trovano ad essere oggetto di una triplice dominazione a causa del genere, della classe, e dell'etnia ${ }^{33}$.

Lavori comunemente definiti sporchi, come il fare le pulizie nelle case, lavare ed accudire gli anziani, cambiare loro il pannolone, portare a spasso il loro cane.... non risultano invitanti per nessuno e le migranti manifestano fortemente la volontà di cambiare lavoro, appena questo sia possibile. Le donne ecuadoriane che ho intervistato hanno ben chiaro che il fatto di svolgere un lavoro così disprezzato dia loro un preciso marchio sociale poiché, come secondo Louis Chevalier, già la rivoluzione industriale aveva creato un nuovo tipo di razzismo differente da quello classico: al gruppo operaio pensato come aggregato omogeneo erano attribuite alcune caratteristiche ritenute innate: la criminalità, il vizio, la sessualità sfrenata ${ }^{34}$... In seguito questo processo di attribuzione si sposta dagli operai ai migranti mettendo in luce come sia, in realtà, il fatto di svolgere un lavoro manuale che dà origine alla razzizazione d'interi gruppi sociali ${ }^{35}$.

\section{FANTASMA EN LA CIUDAD ${ }^{36}$}

Nonostante le asperità della vita in Italia le donne ecuadoriane escludono un ritorno immediato nel paese d'origine, perché ciò equivarrebbe ad ammettere il proprio fallimento presso le comunità di partenza. A causa di ciò alcune di queste donne preferiscono una condizione precaria in Italia piuttosto che il ritorno in Ecuador senza il raggiungimento dei propri obiettivi migratori. Questo accade perché nell'immaginario collettivo ecuadoriano l'Italia è il luogo dove i desideri si possono realizzare, mentre l'Ecuador è quello delle

31 Oso, La inmigración femenina en Madrid y Lisboa, cit. pp. 216-218.

32 Vedi Hannah Arendt, Vita activa. La condizione umana, Milano, Bompiani, 2001.

33 Vedi Giovanna Campani, Genere, etmia e classe, Migrazioni al femminile tra esclusione ed identità. Pisa, ETS, 2000.

34 Etienne Balibar, $l$ razzismo di classe, in Etienne Balibar, Immanuel Wallerstein (a cura di), Razza Nazione Classe, cit. pp. $265-277$, p. 271.

35 Ibidem, pp. 271-274.

36 Dalla canzone Clandestino di Manu Chau. 
aspirazioni frustrate. Le interviste mettono in luce la tensione continua tra il luogo d'origine e quello d'arrivo: pur essendo fisicamente in Italia, le testimoni dichiarano di desiderare di essere in Ecuador. Queste donne sono in equilibrio tra due mondi, o meglio, come afferma una testimone: ni aquí, ni allá.

L'Italia è vista come il luogo del benessere economico, dove le donne possono finalmente permettersi qualcosa più del necessario; il possesso del telefonino appare emblematicamente il simbolo dei desideri realizzati; Le testimoni non sono mai state così esplicite, mi hanno parlato di ciò solo col fluire dell' intervista, quando la sorveglianza attiva sul contenuto dell'intervista si era in qualche modo allentata ${ }^{37}$. Molto spesso questo tipo di motivazione non è riconosciuta nei processi migratori al femminile: comprarsi il telefonino, la casa, dei bei vestiti, permettersi il lusso del più del necessario per vivere rappresenta sempre il lato meno confessabile dei propri desideri, si rischia sempre di essere definite avide dagli appartenenti alla comunità di origine ${ }^{38}$.

L'Ecuador è invece un paese spesso idealizzato, il luogo dove vivono i propri affetti e che ora, magicamente, appare come il paradiso anelato. Questo accade perché anche la memoria è un prodotto in continua costruzione, influenzato dalle circostanze esterne ${ }^{39}$.

La situazione in Italia non è sicuramente delle più rosee; uno degli equivoci relativi alla migrazione ecuadoriana in Europa è che tramite essa molte persone abbiano potuto migliorare la propria condizione ${ }^{40}$. Le donne che ho incontrato hanno difficoltà a definire il tal modo la propria condizione; sono donne del ceto medio, con un altro tasso d'istruzione, o almeno così si presentano, e in Italia svolgono lavoro domestico. Esse dubitano che la propria realizzazione personale ed il proprio status siano migliorati in Italia. In particolare, una testimone mi raccontava che in Ecuador era abituata ad avere persone al proprio servizio, mentre ora è lei stessa ad andare a lavorare a servizio.

E' sempre necessario tenere presente che la migrazione dall'Ecuador è molto recente, perciò molti di coloro che vivono in Italia sono irregolari, come le donne che io ho intervistato.

37 Goffman descrive le maschere che i vari attori sociali indossano a seconda de contesto in cui sono. Vedi: Erving Goffman, La vita quotidiana come rappresentazione, Il Mulino, Bologna, 1969.

38 Francesca Scrinzi, I consumi culturali. I processi di etnicizzazione delle donne marocchine a Genova, in Torre (a cura di), Non sono venuta per scoprire le scarpe, cit. pp. 63-90, pp. 67-68.

39 Vedi Alessandro Portelli, Problemi di metodo, Sulla diversità della storia orale, cit.

40 AA.VV. Verdades y media verdades de la migración, "Cartillas sobre la migración", diciembre del 2002, $n^{\circ} 4$ 
Il ritorno nel paese d'origine, una volta soddisfatti i propri obiettivi, per le ecuadoriane rappresenta il proprio fine.

Difficilmente però il ritorno in Ecuador si realizza, perché, come racconta una testimone, i tempi per la realizzazione del progetto individuale si dilatano enormemente rispetto a ciò che si era pensato in origine: vi è la necessità di guadagnare per ripagare i debiti contratti in Ecuador, ma i primi mesi a Genova passano nella ricerca di un lavoro, e poi si risparmia il denaro per comprare una casa in Ecuador, ma non si può in ogni modo tornare se in quel paese non c'è lavoro.... L'idea del ritorno assume quindi la valenza di un punto di riferimento piuttosto che di una possibilità reale.

\section{CONCLUSIONI}

La politica della chiusura delle frontiere nella cosiddetta Fortezza Europa è determinata dal fatto che l'esigenza dei migranti di una vita differente si contrappone alla necessità economica e politica degli stati di controllare i movimenti della manodopera. Questo è particolarmente evidente dall'analisi condotta sulle donne ecuadoriane, poiché emerge che esse sono accettate se accettano le regole date, se sottostanno alle necessità italiane, cioè se fanno e continueranno a fare le colf o le badanti. L'etnicizzazione dei servizi di cura determina il mantenimento e la creazione di pratiche discriminatorie accentuate dalla condizione di invisibilità in cui le migranti, gran parte delle quali irregolari, si trovano a vivere.

Come emerge dalla descrizioni delle testimoni, non si può prescindere da un'analisi incentrata sulle condizioni materiali nella società sia di partenza che di arrivo; questi due termini di confronto sono strettamente correlati e le proprie percezioni appaiono costantemente modificate dal loro continuo interagire. Il ribadire di avere avuto un certo tenore di vita prima della crisi economica e della conseguente migrazione e il desiderio del ritorno nel paese di origine spiegano anche il forte disagio che le migranti provano nello svolgere lavori di cura. Questo accade poiché, ancora oggi, in molti paesi d'Europa e dell'America Latina, la gestione della casa ricade interamente sul genere femminile, e il lavoro socialmente riconosciuto è quello che viene svolto all'esterno delle mura domestiche.

Comparando le aspettative che le donne ecuadoriane rivestono nella migrazione con il contesto genovese, non si può fare a meno di comprendere la necessità di intraprendere una seria politica 
dell'accoglienza che si confronti con le tematiche espresse dalle migranti: lavoro, abitazione, integrazione con gli italiani...

\section{BIBLIOGRAFIA}

AA. VV. Causas del reciente proceso migratorio ecuadoriano, "Cartilla sobre la migración", diciembre del $2002, \mathrm{n}^{\circ} 3$.

AA.VV. Inmigración femina en el Sur de Europa, Revista de Sociologia, Papers n.60, Universidad Autónoma de Barcelona, 2000.

AA.VV. Verdades y media verdades de la migración, "Cartillas sobre la migración", diciembre del $2002, n^{\circ} 4$.

ACOSTA, A., Ecuador: deuda externa y migración, una relación incestuosa. Texto para la Universidad de GÉnova, 30 abril de 2002.

AMBROSINI, M. Lavorare nell'ombra. L'inserimento degli immigrati nell'economia informale, Fondazione Cariplo-ISMU, 1997.

AMBROSINI, M. Utili invasori. L'inserimento degli immigrati nel mercato del lavoro italiano, Franco-Angeli, Documenti Ismu, Milano, 1999.

AMSELLE, J.L. Logiche meticce. Antropologia dell 'identità in Africa e altrove, Torino, bollati \& Borlinghieri, 1999.

Annuario statistico del Comune di Genova, anni 1998, 1999, 2000.

APPADURAI, A. Modernità in polvere, Roma, Meltemi, 2001.

ARENDT, H. Vita activa. La condizione umana, Milano, Bompiani, 2001.

ARRU, A.; EHMER, J.; RAMELLA, F. (a cura di), Migrazioni, "Quaderni Storici”, anno XXXVI (2001), n. 106, fascicolo 1.

BALIBAR, E.; WALLESRSTEIN, I. Razza nazione e classe, ed. associate, Roma, 1991.

BALIBAR, E. Il razzismo di classe, in Etienne Balibar, Immanuel Wallerstein (a cura di), Razza nazione e classe, ed. associate, Roma, 1991, 265-177.

BELL HOOKS, Elogio del margine, Razza, sesso e mercato culturale, Milano, Feltrinelli, 1998.

BIHR, A. Dall'assalto al cielo all'alternativa, La crisi del movimento operaio europeo, BFS, Pisa, 1995.

BORDO, S. Il peso del corpo, Milano, Feltrinelli, 1997.

CAMPANI, G. Genere, etnia e classe. Migrazioni al femminile tra esclusione ed identità, Pisa, ETS, 2000.

Caritas, Dossier statistico 2000, Roma, Anterem, 2000.

Caritas, Dossier statistico 2003, Roma, Anterem, 2003.

CANCLINI, N.G. Culture ibride, strategie per entrare e uscire dalla modernità, Milano, Angelo Guerrini ed Associati, 1998.

CESCHI, S.; MAZZONI, M. Le forme dello sfruttamento servile e paraschiavistico nel mondo del lavoro, in F. Carchedi, G. Matura, E. Pugliese (a cura di), Il lavoro servile e le nuove serviti,, Milano, Franco Angeli, 2003, 27-45.

CLIFFORD, J. I frutti puri impazziscono. Etnografia, letteratura ed arte nel secolo XX, Torino, Bollati Borlinghieri, 1999.

Colectivo Pro Derechos Humanos PRODH, Derechos Humanos en el Ecuador. Apuntes para el análisis, Ecuador, 2002. 
Consiglio regionale della Liguria, Consulte regionale femminile, Situazioni emergenti di disagio sociale in Liguria. Donne senza dimora, donne immigrate, Atti, Genova, Giovedi 30 settembre 1999.

CORTI, P. Storia delle migrazioni internazionali, Laterza, Roma-Bari, 2003.

DANIELE, G. L'immigrazione terzomondiale a Genova, in Giuliano Carlini (a cura di), La terra in faccia. Gli immigrati raccontano, Roma, Ediesse, 1991, 163-172.

FAVARO, G.; TOGNETTI BORDOGNA, M. Donne dal mondo, strategie migratorie al femminile, Guerini Milano 1991.

FILIPPO, E.; PUGLIESE, E. Le donne nell'immigrazione in Campania, in AA. VV. (a cura di), Inmigración femina en el sur de Europa, Revista de sociologia, papers n.60, Universidad autonoma de Barcellona, 2000, 55-66.

GABACCIA, D. Emigranti, Le diaspore degli italiani dal Medioevo ad oggi, Einaudi, Torino, 2003.

GAMBINO, F. Migranti nella tempesta, Avvistamenti per l'inizio del nuovo millennio, Verona, Ombre corte, 2003.

GOFFMAN, E. La vita quotidiana come rappresentazione, Il Mulino, Bologna, 1969.

GORZ, A. Metamorfosi del lavoro. Critica della ragione economica, Torino, Bollati Boringhieri, 2001.

GRASSO, M. Donne senza confini. Immigrate in Italia tra marginalità ed emancipazione, Torino, l'Harmattan Italia, 1996.

HERRERA, G.; MARTINEZ, A. Género y migración en la region sur, Quito, Facultad Latinoamericana de Ciencias Sociales, 2000.

KOFMAN, E.; PHIZACKLEA, A.; RAGHURAM, P.; SALES, R. Gender and international migration in Europe, Londra, Routlege, 2000.

La Repubblica, venerdi 28 novembre 2003.

MEZZADRA, S. Diritto di fuga, Migrazioni, cittadinanza, globalizzazione, Verona, Ombre corte, 2001.

MORONE, T. "Studi Emigrazione", anno XXXV (1998), n. 130, 317-322.

MOULIER BOUTANG, Y. Dalla schiavitù al lavoro salariato, Manifestolibri, Roma 2003

OSO, L. La inmigración femenina en Madrid y Lisboa: hacia una etnización del servicio doméstico y de las impresas de limpieza, in AA. VV. (a cura di), Inmigración femina en el sur de Europa, Revista de sociologia, papers n.60, Universidad autonoma de Barcellona, 2000, 183-207.

PAGNOTTA, C. Ni aquí, ni allá. L'immigrazione femminile dall'Ecuador a Genova, Tesi di Laurea in Storia, Facoltà di Lettere e Filosofia, anno accademico 2002-2003.

PORTELLI, A. Problemi di metodo, Sulla diversità della storia orale, in C. Bermani (a cura di), Introduzione alla storia orale. Storia, conservazione delle fonti e problemi di metodo, Roma, Odradek, 1999.

Proyecto "Migración, comunicación y desarrollo", El proceso migratorio de ecuatorianos a España, Quito, Centro de Investigaciones ciudad, 2001.

RAUTY, R. Il sogno infranto, la limitazione dell'immigrazione negli Stati Uniti e nelle scienze sociali, Manifestolibri, Roma, 1999.

ROSA, A. Le due società, Torino, Einaudi, 1977.

SAID, E.W. Orientalismo. L'immagine europea dell'Occidente, Milano, Feltrinelli, 1999.

REYNERI, E. La catena migratoria, Bologna, Il Mulino, 1983.

SANFILIPPO M., Problemi di storiografia dell'emigrazione italiana, Sette città, Viterbo, 2002. 
SASSEN, S. Migranti, coloni rifugiati, Dall'emigrazione di massa alla fortezza Europa, Milano, Feltrinelli, 1999.

SAYAD, A. La doppia assenza. Dalle illusioni dell'emigrato alle sofferenze dell'immigrato, Milano, Raffaello Cortina, 2002.

THOMPSON, P. Problemi di metodo nella storia orale, in Luisa Passerini (a cura di), Storia orale. Vita quotidiana e cultura materiale delle classi subalterne, Torino, Rosemberg \& Sellier, 1978, 30-68.

TORRE, A. Il contesto dell 'immigrazione femminile a Genova ed in Liguria, in A. Torre (a cura di), Non sono venuta a scoprire le scarpe. Voci di donne immigrate in Liguria, Dogliani, Sensibili alle foglie, 2001, 9-24.

SEGOVIA, P. Liguria in (a cura di) M. G. Ruggerini, D. Bua, Combattere la discriminazione delle donne migranti, Progetto europeo Codelfi, working paper n.16, luglio $2000 \mathrm{I}$.

SCRINZI, F. I consumi culturali. I processi di etnicizzazione delle donne marocchine a Genova, in A. Torre (a cura di), Non sono venuta per scoprire le scarpe. Voci di donne immigrate in Liguria, Dogliani, Sensibili alle foglie, 2001, 63-90.

WALLERSTEIN, I. Universalismo contro razzismo e sessismo: le tensioni ideologiche del capilalismo, in E. Balibar, I. Wallerstein (a cura di), Razza Nazione Classe. Le identità ambigue, Roma, Edizioni Associate, 1996, 47-56. 